\title{
Continuation of immunosuppressive treatment may be necessary in IgA nephropathy patients with remission of proteinuria: Evaluation by repeat renal biopsy
}

\author{
MIAN-NA LUO*, CUI-WEI YAO*, BI-HUA XU*, YONG-ZHI XU, WEI JING LIU, \\ YONG-MIN FENG, JING-LI TAO and HUA-FENG LIU
}

Institute of Nephrology, Guangdong Medical College, Zhanjiang, Guangdong 524001, P.R. China

Received August 11,2013; Accepted December 23, 2013

DOI: $10.3892 /$ etm.2013.1467

\begin{abstract}
The present study aimed to evaluate the effects of an individualized, low-dose multi-drug immunosuppressive regimen for the treatment of immunoglobulin A nephropathy (IgAN). A preliminary investigation of the course of IgAN following immunosuppressive treatment was conducted based on repeat renal biopsies. Clinical and pathological data of 17 patients with $\operatorname{IgAN}$ who received repeat renal biopsies were analyzed retrospectively. In addition to basic treatment, 16 patients regularly received an individualized low-dose immunosuppressive regimen according to their clinical manifestations and pathological patterns following the first biopsy. Clinical parameters, including 24-h urinary protein excretion and levels of serum albumin, uric acid and total cholesterol were collected. Glomerular deposits of IgA and C3, as well as the activity and chronicity indexes of renal lesions were evaluated by semi-quantitative methods. The 24-h urinary protein excretion of the patients decreased significantly from the first biopsy $(2.53 \pm 2.17 \mathrm{~g} / \mathrm{day})$ to the repeated biopsy $(0.26 \pm 0.55 \mathrm{~g} /$ day $)(\mathrm{P}<0.001)$. Deposits of $\operatorname{IgA}$ and C3 in the glomerulus were persistent, but were reduced in quantity at the second biopsy. Although active renal lesions were observed in the majority of patients, the activity index decreased significantly from $3.18 \pm 1.33$ prior to therapy to $2.47 \pm 0.80$ following therapy $(\mathrm{P}<0.05)$, while the chronicity index did not change significantly $(2.59 \pm 2.00$ versus $2.76 \pm 1.89$, respectively). The individualized, low-dose multi-drug immunosuppressive regimen used in the present study significantly minimized proteinuria, stabilized renal function and alleviated histo-
\end{abstract}

Correspondence to: Professor Hua-Feng Liu, Institute of Nephrology, Guangdong Medical College, 57 Renmin Road, Zhanjiang, Guangdong 524001, P.R. China

E-mail: hf-liu@263.net

*Contributed equally

Key words: course of treatment, immunoglobulin A nephropathy, immunosuppressive treatment, proteinuria, renal pathology logical lesions in patients with $\operatorname{IgAN}$ without causing overt adverse effects during the short-term follow-up. In addition to proteinuria, renal pathological changes should be appraised when considering the withdrawal of immunosuppressants from $\operatorname{Ig} \mathrm{AN}$ treatment.

\section{Introduction}

Immunoglobulin A nephropathy ( $\operatorname{IgAN})$, an immune complex-mediated glomerulonephritis, is the most common form of primary glomerulonephritis worldwide and is characterized by deposits of $\operatorname{IgA}$ as either the dominant or codominant immunoglobulin $(1,2)$. Proteinuria is regarded as a risk factor for an unfavorable renal prognosis and the reduction of proteinuria is considered an important therapeutic goal in clinical practice (3-5). In addition to proteinuria, active renal lesions, such as cellular crescent formation, diffuse mesangial proliferation and interstitial inflammatory infiltration have been identified to result in a rapid rate of deterioration and lower kidney survival (6).

At present, the optimal immunosuppressive strategy for IgAN treatment, particularly the duration of treatment, remains unclear. Treatment with glucocorticoids alone or combined therapy with glucocorticoids and other immunosuppressive agents have shown beneficial effects by reducing proteinuria and improving renal function $(7,8)$. According to the guidelines 'Kidney Disease: Improving Global Outcomes' (KDIGO) (9), which are based on the results of previous studies $(7,10,11)$, patients with persistent proteinuria of $>1 \mathrm{~g}$ /day following 6 months of treatment with renin-angiotensin system (RAS) inhibitors are suggested to receive a 6-month course of corticosteroid therapy. However, certain issues require clarification, including whether immunomodulatory treatment should be continued or withdrawn in patients with proteinuria of $<1 \mathrm{~g} /$ day following 6 months of corticosteroid treatment, and whether the complete resolution of proteinuria is equivalent to the disappearance of renal active lesions. Furthermore, the effectiveness of immunosuppressive regimens other than glucocorticoid monotherapy for this heterogeneous disease require investigation

In the present study, the effects of an individualized, low-dose multi-drug immunosuppressive regimen on $\operatorname{IgAN}$ 
Table I. Baseline characteristics of 17 patients with IgA nephropathy who underwent a repeat renal biopsy.

\begin{tabular}{|c|c|c|c|c|c|}
\hline Patient & Gender & $\begin{array}{c}\text { Age at first } \\
\text { biopsy (years) }\end{array}$ & $\begin{array}{l}\text { Interval } \\
\text { (months) }\end{array}$ & $\begin{array}{l}\text { RAS } \\
\text { inhibitors }\end{array}$ & $\begin{array}{c}\text { Immunosuppressive } \\
\text { treament }\end{array}$ \\
\hline 1 & $\mathrm{~F}$ & 28 & 12 & Yes & $\mathrm{PDN}+\mathrm{TwHF}+\mathrm{AZA}$ \\
\hline 2 & M & 21 & 50.5 & Yes & $\mathrm{PDN}+\mathrm{TwHF}+\mathrm{AZA}$ \\
\hline 3 & M & 18 & 9 & Yes & $\mathrm{PDN}+\mathrm{TwHF}+\mathrm{CTX} \rightarrow \mathrm{AZA}$ \\
\hline 4 & M & 19 & 15 & Yes & $\mathrm{PDN}+\mathrm{TwHF}+\mathrm{AZA}$ \\
\hline 5 & M & 14 & 15 & Yes & $\mathrm{PDN}+\mathrm{TwHF}+\mathrm{AZA}$ \\
\hline 6 & $\mathrm{~F}$ & 59 & 21 & Yes & $\mathrm{PDN}+\mathrm{TwHF}$ \\
\hline 7 & M & 22 & 17.5 & Yes & $\mathrm{PDN}+\mathrm{TwHF}+\mathrm{AZA}$ \\
\hline 8 & M & 61 & 12.5 & Yes & $\mathrm{PDN}+\mathrm{TwHF}+\mathrm{AZA}$ \\
\hline 9 & $\mathrm{~F}$ & 31 & 14 & Yes & $\mathrm{PDN}+\mathrm{TwHF}+\mathrm{AZA}$ \\
\hline 10 & $\mathrm{~F}$ & 39 & 14 & Yes & $\mathrm{PDN}+\mathrm{TwHF}+\mathrm{AZA}$ \\
\hline 11 & M & 26 & 12.5 & Yes & $\mathrm{PDN}+\mathrm{TwHF}+\mathrm{AZA}$ \\
\hline 12 & M & 35 & 15 & Yes & PDN \\
\hline 13 & M & 14 & 11 & Yes & $\mathrm{PDN}+\mathrm{AZA}$ \\
\hline 14 & $\mathrm{~F}$ & 32 & 16.6 & Yes & $\mathrm{PDN}+\mathrm{TwHF}+\mathrm{CTX} \rightarrow \mathrm{AZA}$ \\
\hline 15 & M & 26 & 17 & Yes & $\mathrm{PDN}+\mathrm{TwHF}+\mathrm{AZA}$ \\
\hline 16 & M & 55 & 12 & Yes & $\mathrm{PDN}+\mathrm{AZA}$ \\
\hline 17 & $\mathrm{~F}$ & 18 & 11.5 & Yes & $\mathrm{PDN}+\mathrm{TwHF}$ \\
\hline
\end{tabular}

IgA, immunoglobulin A; RAS, renin-angiotensin system; PDN, prednisone; TwHF, Tripterygium wilfordii Hook F; AZA, azathioprine; and CTX, cyclophosphamide.

treatment were retrospectively evaluated and a preliminary investigation of the duration of immunosuppressive treatment for IgAN based on repeat renal biopsies was conducted.

\section{Materials and methods}

Data collection. Clinical data of 17 patients diagnosed with primary $\operatorname{IgAN}$ by biopsy, including 11 males and 6 females with a mean age of 30.5 years (range, 14-61 years) were collected from the medical records at The Affiliated Hospital of Guangdong Medical College (Zhangjiang, China). All patients were notified and agreed for their clinical data to be used in this study. The institutional review board of the Affiliated Hospital of Guangdong Medical College approved this study and waived the requirement for patient consent.

Therapeutic intervention. None of the patients received steroids or other immunosuppressive agents prior to the first renal biopsy. As shown in Table I, following the first biopsy, 16 patients were regularly treated with low-dose prednisone (PDN) alone or in combination with one or two other low-dose immunosuppressants. One patient (patient no. 2) discontinued immunosuppressive therapy from months 9 to 28 . Generally, the immunosuppressive regimen was individualized according to the patient's clinical and pathological presentation at the first biopsy. Briefly, in severe cases of proteinuria and/or the marked presence of active renal lesions the high dose immunosuppressants were administered. The initial dose of PDN was $\sim 0.5 \mathrm{mg} /(\mathrm{kg} \cdot$ day $)$ for 8 weeks followed by a $10 \%$ reduction of the original dose in 1 month and gradual tapering to maintain a dose of
5-10 mg/day. Tripterygium wilfordii Hook F (TwHF) was administered orally at an initial dose of $\sim 1.0 \mathrm{mg} /(\mathrm{kg} \cdot \mathrm{day})$ in three divided doses for $\sim 6$ months and gradually tapered to $20-30 \mathrm{mg} /$ day as the maintenance therapy. Azathioprine (AZA) was administered at a single dose of $1-2 \mathrm{mg} /(\mathrm{kg} \cdot$ day) for $\sim 6$ months and gradually tapered to $25-50 \mathrm{mg}$ /day as the maintenance therapy. Intravenous cyclophosphamide (CTX) was administered at 0.8-1.0 g per month for 3 months, followed by $1-2 \mathrm{mg} /(\mathrm{kg} \cdot$ day) AZA for an additional 3 months and then gradually tapered to $25-50 \mathrm{mg} /$ day. All patients received angiotensin-converting-enzyme inhibitor (ACEI) and/or angiotensin II receptor blocker (ARB) as part of their anti-hypertensive regimen or their basic therapy. Six patients (patient nos. 4, 6, 9, 11, 12 and 14) received lipid-lowering agents due to the presence of hyperlipidemia and 8 patients (patient nos. 1, 2, 3, 4, 5, 7, 10 and 11) received allopurinol due to hyperuricemia.

Follow-up. Sixteen patients underwent regular follow-ups at the outpatient clinic approximately once a month until they received the second biopsy. Patient no. 2 discontinued follow-up from months 9 to 28 but continued from months 28 to 50.5. During the follow-up, the clinical symptoms, drug consumption and possible treatment complications of the patients were evaluated. Blood pressure was monitored and standard body examinations were performed. Laboratory parameters, including serum levels of creatinine, uric acid, cholesterol and albumin, as well as results of routine blood tests were recorded. Every month, urinary protein was semiquantified. The 24-h urinary protein excretion level was measured at the first and second renal biopsies. The glomerular 
A

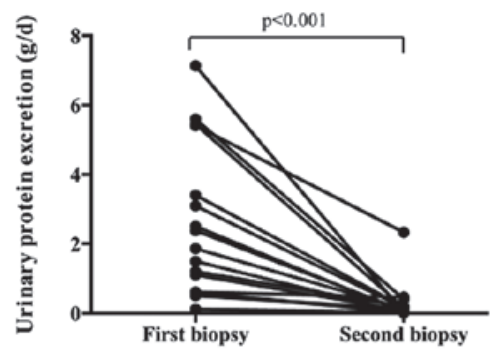

D

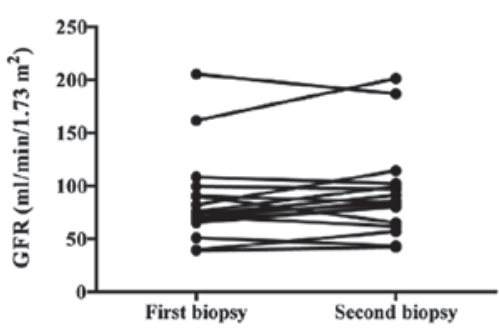

B

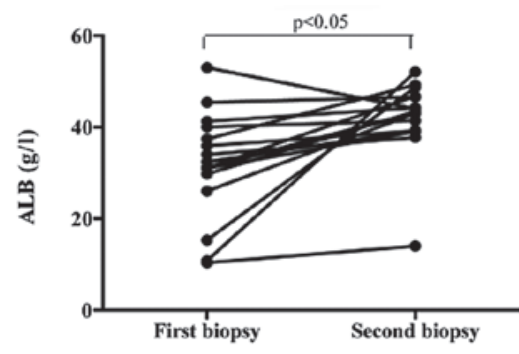

$\mathbf{E}$

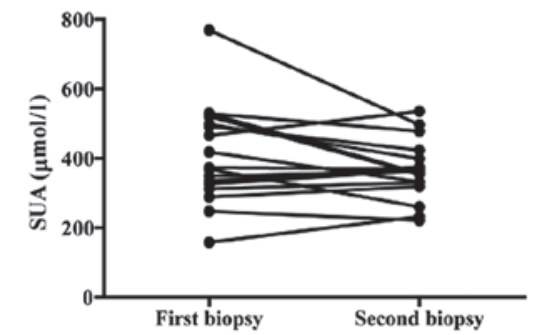

C

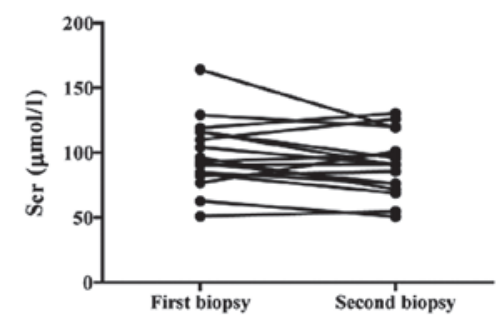

$\mathbf{F}$

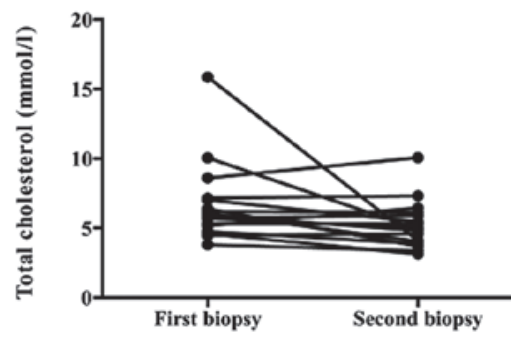

Figure 1. Comparison of the clinical and pathological data of 17 patients with IgAN at the first and second renal biopsies. (A) Urinary protein excretion decreased significantly from $2.53 \pm 2.17 \mathrm{~g} /$ day at the first biopsy to $0.26 \pm 0.55 \mathrm{~g} / \mathrm{day}$ at the second biopsy $(\mathrm{P}<0.001)$; (B) ALB increased from $31.54 \pm 11.34 \mathrm{~g} / 1$ at the first biopsy to $42.06 \pm 8.24 \mathrm{~g} / 1$ at the second biopsy $(\mathrm{P}<0.05$ ); (C) Scr decreased from $98.29 \pm 26.51 \mu \mathrm{mol} / 1$ at the first biopsy to $91.00 \pm 23.54 \mu \mathrm{mol} / 1$ at the second biopsy ( $\mathrm{P}>0.05$ ); and (D) GFR increased from $86.14 \pm 41.77 \mathrm{ml} / \mathrm{min} / 1.73 \mathrm{~m}^{2}$ at the first biopsy to $92.62 \pm 43.13 \mathrm{ml} / \mathrm{min} / 1.73 \mathrm{~m}{ }^{2}$ at the second biopsy $(P>0.05)$. GFR was calculated using the modification of diet in renal disease formula. (E) SUA and (F) total cholesterol levels were not significantly different between the two biopsies though both levels were reduced at the second biopsy in comparison with those at the first biopsy. IgAN, immunoglobulin A nephropathy; ALB, serum albumin; Scr, mean serum creatinine; GFR, glomerular filtration rate; SUA, serum uric acid.

filtration rate (GFR) was estimated using the Modification of Diet in Renal Disease study (MDRD) formula: [eGFR (ml/ $\left.\min / 1.73 \mathrm{~m}^{2}\right)=186 * \mathrm{Scr}(\mathrm{mg} / \mathrm{dl})-1.154 *$ age $($ years $)-0.203$ * (0.742 femal].

Renal biopsies and pathological diagnosis. All patients underwent renal biopsies twice with an interval time of 16.2 \pm 9.3 months (range, 9.0-50.5 months) under ultrasound guidance. Following hematoxylin and eosin, Masson's trichrome, silver methenamine, periodic acid-Schiff and immunofluorescent staining, the renal specimens were assessed by one renal pathologist and one clinical nephrologist who were blinded to the clinical information. In immunofluorescent staining, the deposition of $\operatorname{IgA}, \operatorname{IgG}, \operatorname{IgM}$ and complement C3 was semi-quantified as follows: Negative, (-); minimal in intensity, $( \pm)$; slight in intensity, (1+); moderate in intensity, $(2+)$; marked in intensity, (3+); and marked in intensity and extent, (4+). These semi-quantified results were converted into scores as follows: $0,(-) ; 0.5,( \pm) ; 1,(1+) ; 2,(2+) ; 3,(3+)$; and $4(4+)$, respectively. The activity and chronicity indices were evaluated as previously described (12). Briefly, the activity index was first graded for mesangial proliferation (grades 0-3: Normal, 0; slight, 1; moderate, 2; and severe, 3), interstitial inflammatory cell infiltration (grades $0-3$ : None, $0 ; 1-20 \%$, $1 ; 21-50 \%, 2$; and $>50 \%, 3$ ) and cellular crescent formation (grades 0-3 according to the percentage of glomeruli involved in crescents: None, $0 ; 1-20 \%, 1 ; 21-50 \%, 2$; and $>50 \%, 3)$. The sum of these scores was subsequently computed (maximum of 9). The chronicity index was first graded for the percentage of glomeruli exhibiting fibrous crescents (grades 0-3: None, 0; $1-20 \%, 1 ; 21-50 \%, 2$; and $>50 \%, 3$ ), the percentage of glomeruli exhibiting segmental or global sclerosis (grades 0-3: None, 0; $1-20 \%, 1 ; 21-50 \%, 2$; and $>50 \%, 3$ ), and the degrees of tubular atrophy (on a scale of 0-3) and interstitial fibrosis (on a scale of 0-3). The sum of these scores was subsequently computed (maximum of 12).

Statistical analysis. Statistical tests were performed with SPSS software, version 16.0 for Windows (SPSS Inc., Chicago, IL, USA). Normally distributed variables were assessed using the paired-samples t-test. Nonparametric variables and non-normally distributed variables were assessed by Wilcoxon signed rank test. $\mathrm{P}<0.05$ was considered to indicate a statistically significant difference.

\section{Results}

General data. The regimen was well tolerated and no severe adverse events were observed. Blood pressure in all patients was controlled to the standard of $\sim 130 / 80 \mathrm{mmHg}$ during the course of follow-up. According to renal pathological diagnosis of the second biopsy, five patients (patient nos. 5, 10, 14, 15 and 17) were able to discontinue immunosuppressive treatment as substantially no active renal lesions were observed, while the remaining patients continued the regimen with a very low dose of immunosuppressants due to existing residual active renal lesions.

Clinical data. Semi-quantified analysis showed that the urinary protein levels in all patients decreased gradually to normal levels from months 2 to 7 (data not shown). The mean 24-h protein excretion levels markedly declined from $2.53 \pm 2.17 \mathrm{~g} / \mathrm{day}$ at the first biopsy to $0.26 \pm 0.55 \mathrm{~g} /$ day at the second biopsy (patient no. 2 was $2.33 \mathrm{~g} /$ day) $(\mathrm{P}<0.001$; Fig. 1A). Correspondingly, the serum albumin levels increased significantly from $31.5 \pm 11.3$ to $42.1 \pm 8.2 \mathrm{~g} / \mathrm{l}(\mathrm{P}<0.05$; Fig. $1 \mathrm{~B})$. The serum creatinine levels decreased from $98.3 \pm 26.5$ to 91.0 $\pm 23.5 \mu \mathrm{mol} / 1$ (Fig. 1C), while the GFR increased from 
Table II. Histological data of 17 patients with IgAN who underwent a repeat renal biopsy.

\begin{tabular}{|c|c|c|c|c|c|c|}
\hline \multirow[b]{2}{*}{ Patient } & \multirow[b]{2}{*}{ Biopsies } & \multicolumn{2}{|c|}{ Intensity of deposits } & \multicolumn{3}{|c|}{ Index of renal pathological lesions } \\
\hline & & $\operatorname{Ig} \mathrm{A}$ & $\mathrm{C} 3$ & AS & $\mathrm{CS}$ & TS \\
\hline \multirow[t]{2}{*}{1} & First & 3 & 3 & 5 & 4 & 9 \\
\hline & Second & 2 & 2 & 4 & 2 & 6 \\
\hline \multirow[t]{2}{*}{2} & First & 2 & 0 & 2 & 0 & 2 \\
\hline & Second & 2 & 0 & 3 & 6 & 9 \\
\hline \multirow[t]{2}{*}{3} & First & 1 & 4 & 4 & 5 & 9 \\
\hline & Second & 1 & 1 & 2 & 3 & 5 \\
\hline \multirow[t]{2}{*}{4} & First & 1 & 0 & 2 & 1 & 3 \\
\hline & Second & 0.5 & 0 & 1 & 0 & 1 \\
\hline \multirow[t]{2}{*}{5} & First & 3 & 0.5 & 2 & 0 & 2 \\
\hline & Second & 2 & 0.5 & 3 & 1 & 4 \\
\hline \multirow[t]{2}{*}{6} & First & 4 & 4 & 3 & 5 & 8 \\
\hline & Second & 4 & 1 & 2 & 6 & 8 \\
\hline \multirow[t]{2}{*}{7} & First & 3 & 2 & 3 & 4 & 7 \\
\hline & Second & 3 & 2 & 3 & 3 & 7 \\
\hline \multirow[t]{2}{*}{8} & First & 3 & 2 & 6 & 3 & 9 \\
\hline & Second & 3 & 2 & 2 & 3 & 5 \\
\hline \multirow[t]{2}{*}{9} & First & 4 & 2 & 2 & 0 & 2 \\
\hline & Second & 2 & 1 & 2 & 4 & 6 \\
\hline \multirow[t]{2}{*}{10} & First & 3 & 2 & 5 & 5 & 10 \\
\hline & Second & 2 & 2 & 4 & 6 & 10 \\
\hline \multirow[t]{2}{*}{11} & First & 4 & 3 & 5 & 1 & 6 \\
\hline & Second & 2 & 2 & 3 & 1 & 4 \\
\hline \multirow[t]{2}{*}{12} & First & 2 & 0.5 & 2 & 1 & 3 \\
\hline & Second & 2 & 0 & 2 & 2 & 4 \\
\hline \multirow[t]{2}{*}{13} & First & 4 & 3 & 3 & 3 & 6 \\
\hline & Second & 3 & 3 & 2 & 2 & 4 \\
\hline \multirow[t]{2}{*}{14} & First & 2 & 0.5 & 2 & 6 & 8 \\
\hline & Second & 1 & 0 & 2 & 2 & 4 \\
\hline \multirow[t]{2}{*}{15} & First & 3 & 2 & 2 & 2 & 4 \\
\hline & Second & 3 & 2 & 2 & 1 & 3 \\
\hline \multirow[t]{2}{*}{16} & First & 3 & 0 & 3 & 3 & 6 \\
\hline & Second & 1 & 0 & 3 & 4 & 7 \\
\hline \multirow[t]{2}{*}{17} & First & 3 & 3 & 3 & 1 & 4 \\
\hline & Second & 2 & 2 & 2 & 1 & 3 \\
\hline
\end{tabular}

IgAN, immunoglobulin A nephropathy; AS, activity index score; CS, chronicity index score; TS, total score. The intensity of deposits detected by immunofluorescent microscopy was graded semi-quantitatively on a scale from 0 to 4 : Negative, 0 ; minimal in intensity, 0.5 ; slight in intensity, 1 ; moderate in intensity, 2; marked in intensity, 3 ; and marked in intensity and extent, 4.

$86.1 \pm 41.8$ to $92.6 \pm 43.1 \mathrm{ml} / \mathrm{min} / 1.73 \mathrm{~m}^{2}$ (Fig. 1D); however, there were no significant differences in these levels between the two biopsies. Additionally, serum uric acid and total cholesterol levels were not significantly different between the two biopsies (Fig. 1E and F).

Notably, proteinuria decreased gradually to normal level at 5 months in patient no. 2 who received the combined therapy with a low-dose of PDN, TwHF and AZA. The patient discontinued immunosuppressive treatment at 9 months and proteinuria relapsed severely at 28 months. Although immunosuppressants were administered again following relapse, proteinuria was not alleviated until the second biopsy at 50.5 months.

Pathological data. The intensity of glomerular IgA and C3 deposits was dramatically decreased at the second biopsy compared with that at the first biopsy $(\mathrm{P}=0.004$ and $\mathrm{P}=0.011$, respectively); however, glomerular IgA and/or C3 deposits remained present in the majority of patients at the second biopsy (Table II). The intensity of other immunoglobulin deposition was not significantly different between the two biopsies (data not shown). The acute lesions, such as diffuse mesangial 

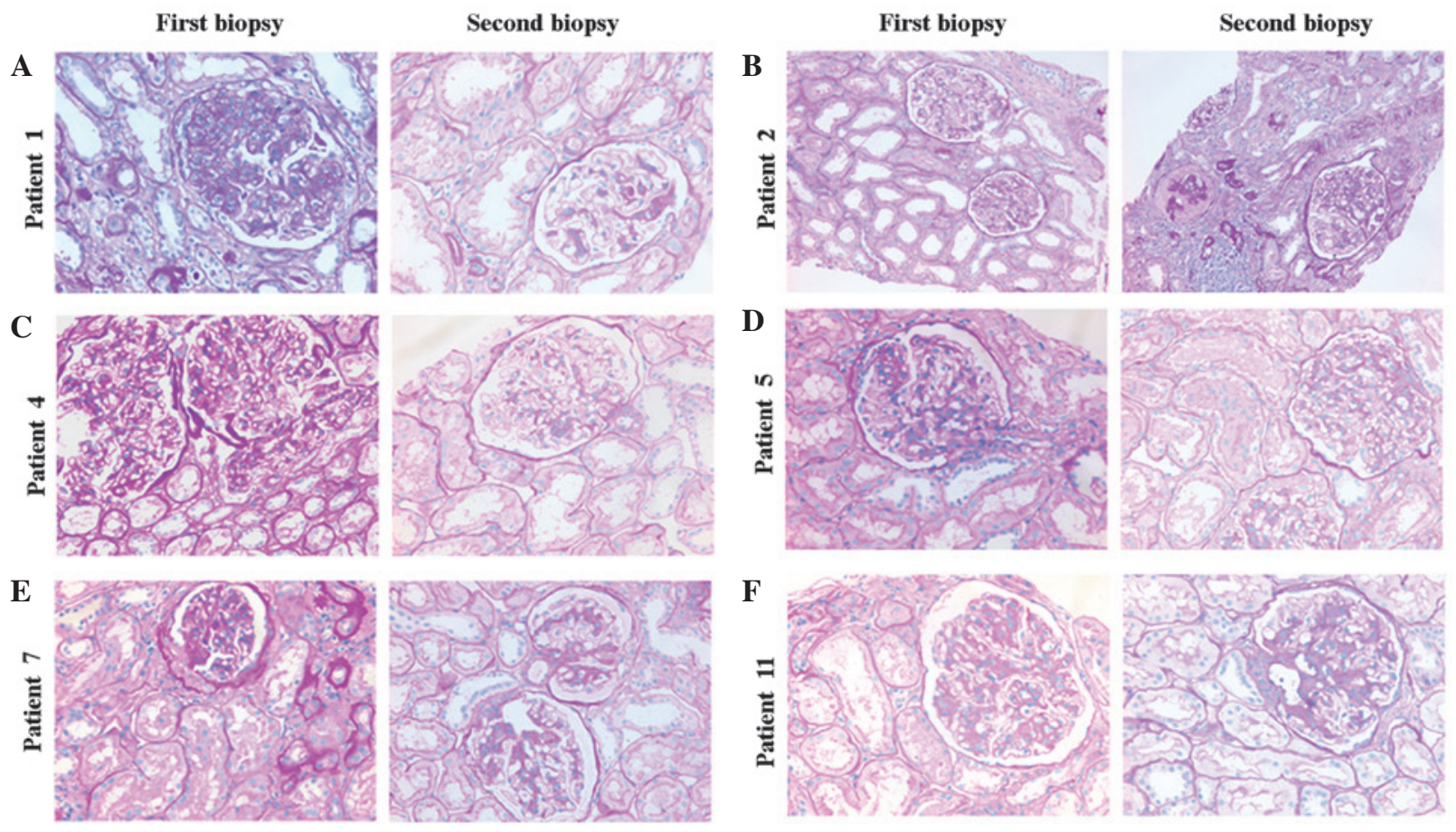

Figure 2. Representative histological sections following immunomodulatory therapy in immunoglobulin A nephropathy. All sections were stained with periodic acid-Schiff. (A) In patient 1, at the first renal biopsy moderate diffuse mesangial cell proliferation, increased matrix deposition, adhesion to the Bowman's capsule and interstitial mononuclear cell infiltration were observed. At the second renal biopsy, slight mesangial cell proliferation and matrix expansion, but no adhesion to the Bowman's capsule or interstitial mononuclear cell infiltration were observed. (B) In patient 2, compared with that at the first biopsy, segmental glomerulosclerosis, interstitial infiltration, tubular atrophy and interstitial fibrosis were observed in the second renal biopsy. The histopathological features had deteriorated at the second renal biopsy (magnification, $\mathrm{x} 200$ ). (C,D,F) In patients 4, 5 and 11, the mesangial proliferation and matrix increases observed in the first biopsy were ameliorated at the second renal biopsy. No major changes of tubulointerstitial lesions were visible (magnification, $\mathrm{x} 400$ ). (E) In patient 7 , at the first renal biopsy, glomerular cell proliferation, matrix expansion, focal adhesion to the Bowman's capsule, tubular atrophy and interstitial fibrosis were observed. At the second renal biopsy, mild mesangial cell proliferation and adhesion to the Bowman's capsule were detected. (A,C-F) Histopathological changes were improved at the second renal biopsy (magnification, $\mathrm{x} 400$ ).

proliferation, cellular crescent formation or interstitial mononuclear cell infiltrates showed marked amelioration in the majority of patients at the second biopsy compared with that at the first (Fig. 2). In addition, chronic pathological damage, including glomerular capsular adhesion, segmental glomerular sclerosis, global sclerosis and the degree of interstitial fibrosis, did not show progression in the majority of patients (Fig. 2). As indicated by the results in Table II, the activity index decreased significantly from $3.18 \pm 1.33$ to $2.47 \pm 0.80$ $(\mathrm{P}<0.05)$, while the chronicity index remained unchanged $(2.59 \pm 2.00$ versus $2.76 \pm 1.89)$. Moreover, although the score of acute lesions decreased, residual renal active lesions were identified in 12 patients who had almost complete remission of proteinuria at the second renal biopsy. Histopathology was aggravated severely in patient no. 2, observed as severe glomerular sclerosis and interstitial fibrosis (Fig. 2B).

\section{Discussion}

The benefits of corticosteroid monotherapy for the treatment of $\operatorname{IgAN}$ have been elucidated in several studies $(7,13,14)$ and suggested in the KDIGO guidelines. However, an additional randomly controlled trial conducted in Japan showed that corticosteroid monotherapy was not beneficial for protecting kidney function (15). AZA is not suggested for the treatment of IgAN according to the KDIGO guidelines; however, the addition of AZA to corticosteroids has been shown to provide further benefits in the reduction of proteinuria and the stabilization of renal function in patients with $\operatorname{IgAN}$, particularly in those who did not respond to corticosteroid monotherapy (16). Notably, Shima et al showed that glomerular IgA deposits disappeared following combined therapy with corticosteroids and AZA in children with IgAN showing diffuse mesangial proliferation (17). TwHF, an extract from a traditional Chinese medicine, has been widely used to treat autoimmune and inflammatory diseases, including lupus nephritis and rheumatoid arthritis (18-20). A previous study showed that Chinese patients with IgAN benefited from TwHF treatment (21). In conclusion, the optimal treatment strategy for IgAN remains controversial, despite the KIDIGO guidelines.

In the past few years, prior to the publication of the KDIGO guidelines for IgAN treatment, we applied an individualized, low-dose and multi-drug immunosuppressive regimen for the treatment of IgAN based on clinical and pathological patterns, which was inspired from the idea of multi-target therapy for lupus nephritis (22). All patients in the present retrospective study received relatively low-dose PDN treatment in order to avoid the obvious side-effects of high-dose steroids. However, to compensate for the insufficient immunosuppressive strength of low-dose PDN, AZA or a short course of CTX was administered to those patients with more severe proteinuria and/or active pathological changes. The dosage was flexibly adjusted according to the condition of the patient. In particular, the traditional Chinese medicine, TwHF, was also added to the two 
immunosuppressants in the majority of patients, which formed a triple immunosuppressive regimen. Notably, it was observed that this immunosuppressive regimen significantly decreased proteinuria, increased serum albumin levels and stabilized renal function. Complete remission of proteinuria was achieved in the majority of patients. However, it may be suggested that basic therapy, such as ACEI and/or ARB usage and blood pressure control may have also contributed to the reduction in proteinuria. However, according to previous studies, basic RAS inhibition fails to achieve complete remission of proteinuria in the majority of patients with $\operatorname{IgAN}(23,24)$, which suggests that immunosuppressive treatment may be important for proteinuria remission in the patients in the present study. Furthermore, a decline in the renal pathological activity index indicated that renal inflammation was controlled at the second biopsy. The chronicity index did not increase in the majority of patients, suggesting that no additional active renal lesions developed into chronic destruction. It has been suggested that the intensity of glomerular IgA and C3 deposition may be associated with $\operatorname{IgAN}$ progression $(25,26)$ and the disappearance of $\operatorname{Ig} \mathrm{A}$ indicates a better prognosis (17). In the present study, the quantities of $\operatorname{Ig} \mathrm{A}$ and $\mathrm{C} 3$ deposits were significantly decreased, indicating that the remission of proteinuria and improvement of renal pathological lesions may be due to the minimizing of the abnormal immune inflammatory response by the combined immunosuppressive strategy, and may be independent to the basic therapy. This supports the positive therapeutic effects of this immunosuppressive regimen on IgAN. In addition, the side-effects were mild and patients successfully tolerated the immunosuppressive regimen.

According to the KDIGO guidelines, the duration of corticosteroid treatment is 6 months in patients with IgAN and proteinuria of $>1 \mathrm{~g} /$ day. However, the present study indicated that although proteinuria was markedly decreased (as shown in Fig. 1A), the deposits of $\operatorname{IgA}$ and C3 and renal active lesions remained in the glomerulus following combined immunosuppressive treatment for $\sim 1$ year in the majority of patients. This indicated that complete remission of proteinuria did not conform to the disappearance of histopathological activity. Thus, a 6-month course of immunosuppressive therapy may not be enough to completely control renal active lesions and a longer course of treatment may be required for certain patients with IgAN. Moreover, in patient no. 2, proteinuria relapsed following the withdrawal of immunosuppressants. The patient had not achieved complete remission of proteinuria and chronic histopathological changes with significant deterioration were observed at the second renal biopsy, which may have resulted from failure to continue treatment. Therefore, remission of proteinuria may not be the only decisive factor for withdrawing immunosuppressants in certain patients with IgAN and additional factors, such as renal pathology should be considered $(12,27)$.

It is widely considered that proteinuria of $<1 \mathrm{~g} /$ day indicates a favorable prognosis (3) and such patients require no specific treatment, but should be kept under periodic review $(28,29)$. However, Usui et al (30) demonstrated that certain patients with mild proteinuria $(<0.5 \mathrm{~g} /$ day) progress to dialysis. In the present study, five patients with an initial proteinuria of $<1 \mathrm{~g} /$ day presented marked active renal lesions at the first biopsy and were administered a very low-dose of the multi-drug immunosuppressive regimen for $\sim 1$ year. During follow-up, the patients achieved complete remission of proteinuria and their renal pathological lesions improved. This supports the idea that immunosuppressive therapy will bring additional benefits for those patients with IgAN whose urinary protein is $<1 \mathrm{~g} /$ day, but show existing active renal lesions.

It should be acknowledged that there are limitations in the present study, including the lack of a control group, a small sample size, a retrospective study design and the relatively short follow-up period. Therefore, the optimal drug-combined regimen and duration of immunosuppressive therapy in patients with $\operatorname{Ig} \mathrm{AN}$ requires further study.

In conclusion, the individualized low-dose multi-drug immunosuppressive regimen significantly minimized proteinuria, stabilized renal function and alleviated histological lesions without inducing overt adverse effects in patients with $\mathrm{IgAN}$ in the short-term follow-up. However, optimal treatment strategies require further investigation. Notably, the results also indicated that additional factors to proteinuria, particularly renal pathological alterations, should be considered when withdrawing immunosuppressant therapy from IgAN treatment. The duration of immunosuppressive therapy on $\operatorname{Ig} \mathrm{AN}$ requires further study.

\section{Acknowledgements}

This work was supported by Zhanjing city program for tackling key problems in science and technology (No. 2013B01296) and Youth Foundation of the Affiliated Hospital of Guangdong Medical College (No. QK1301).

\section{References}

1. Floege $\mathrm{J}$ and Feehally J: $\operatorname{Ig} A$ nephropathy: recent developments. J Am Soc Nephrol 11: 2395-2403, 2000.

2. Donadio JV and Grande JP: IgA nephropathy. N Engl J Med 347: 738-748, 2002.

3. Reich HN, Troyanov S, Scholey JW and Cattran DC; Toronto Glomerulonephritis Registry: Remission of proteinuria improves prognosis in IgA nephropathy. J Am Soc Nephrol 18: 3177-3183, 2007.

4. D'Amico G: Natural history of idiopathic IgA nephropathy and factors predictive of disease outcome. Semin Nephrol 24: 179-196, 2004.

5. Berthoux F, Mohey H, Laurent B, Mariat C, Afiani A and Thibaudin L: Predicting the risk for dialysis or death in IgA nephropathy. J Am Soc Nephrol 22: 752-761, 2011.

6. Coppo R, Cattran D, Roberts Ian SD, et al: The new Oxford Clinico-Pathological Classification of $\operatorname{IgA}$ nephropathy. Prilozi 31: 241-248, 2010.

7. Pozzi C, Bolasco PG, Fogazzi GB, et al: Corticosteroids in IgA nephropathy: a randomised controlled trial. Lancet 353: 883-887, 1999.

8. Harmankaya O, Oztürk Y, Baştürk T, Obek A and Kiliçarslan I: Efficacy of immunosuppressive therapy in IgA nephropathy presenting with isolated hematuria. Int Urol Nephrol 33: 167-171, 2002.

9. KDIGO: Kidney Disease: Improving Global Outcomes. KDIGO Clinical Practice Guideline for Glomerulonephritis. Kidney Int 2 (suppl): 209-217, 2012

10. Manno C, Torres DD, Rossini M, Pesce F and Schena FP: Randomized controlled clinical trial of corticosteroids plus ACE-inhibitors with long-term follow-up in proteinuric IgA nephropathy. Nephrol Dial Transplant 24: 3694-3701, 2009.

11. Lv J, Zhang H, Chen Y, et al: Combination therapy of prednisone and ACE inhibitor versus ACE-inhibitor therapy alone in patients with IgA nephropathy: a randomized controlled trial. Am J Kidney Dis 53: 26-32, 2009.

12. Andreoli SP and Bergstein JM: Treatment of severe IgA nephropathy in children. Pediatr Nephrol 3: 248-253, 1989. 
13. Tamura S, Ueki K, Ideura H, et al: Corticosteroid therapy in patients with IgA nephropathy and impaired renal function. Clin Nephrol 55: 192-195, 2001.

14. Moriyama T, Honda K, Nitta K, Yumura W and Nihei H: The effectiveness of steroid therapy for patients with advanced $\operatorname{Ig} \mathrm{A}$ nephropathy and impaired renal function. Clin Exp Nephrol 8: 237-242, 2004

15. Katafuchi R, Ikeda K, Mizumasa T, et al: Controlled, prospective trial of steroid treatment in IgA nephropathy: a limitation of low-dose prednisolone therapy. Am J Kidney Dis 41: 972-983, 2003.

16. Stangou M, Ekonomidou D, Giamalis P, et al: Steroids and azathioprine in the treatment of IgA nephropathy. Clin Exp Nephrol 15: 373-380, 2011

17. Shima Y, Nakanishi K, Kamei K, et al: Disappearance of glomerular IgA deposits in childhood IgA nephropathy showing diffuse mesangial proliferation after 2 years of combination/prednisolone therapy. Nephrol Dial Transplant 26: 163-169, 2011.

18. Qiu D and Kao PN: Immunosuppressive and anti-inflammatory mechanisms of triptolide, the principal active diterpenoid from the Chinese medicinal herb Tripterygium wilfordii Hook. f. Drugs R D 4: 1-18, 2003.

19. Lipsky PE and Tao XL: A potential new treatment for rheumatoid arthritis: thunder god vine. Semin Arthritis Rheum 26: 713-723, 1997.

20. Tao X and Lipsky PE: The Chinese anti-inflammatory and immunosuppressive herbal remedy Tripterygium wilfordii Hook F. Rheum Dis Clin North Am 26: 29-50, 2000.

21. Chen YZ, Gao Q, Zhao XZ, et al: Meta-analysis of Tripterygium wilfordii Hook $\mathrm{F}$ in the immunosuppressive treatment of $\mathrm{IgA}$ nephropathy. Intern Med 49: 2049-2055, 2010.
22. Bao H, Liu ZH, Xie HL, Hu WX, Zhang HT and Li LS: Successful treatment of class V+IV lupus nephritis with multitarget therapy. J Am Soc Nephrol 19: 2001-2010, 2008.

23. Cheng J, Zhang X, Tian J, Li Q and Chen J: Combination therapy an ACE inhibitor and an angiotensin receptor blocker for IgA nephropathy: a meta-analysis. Int J Clin Pract 66: 917-923, 2012.

24. Cheng J, Zhang W, Zhang XH, He Q, Tao XJ and Chen JH: ACEI/ARB therapy for IgA nephropathy: a meta analysis of randomised controlled trials. Int J Clin Pract 63: 880-888, 2009.

25. van Dixhoorn MG, Sato T, Muizert Y, van Gijlswijk-Janssen DJ, De Heer E and Daha MR: Combined glomerular deposition of polymeric rat IgA and $\operatorname{IgG}$ aggravates renal inflammation. Kidney Int 58: 90-99, 2000

26. Komatsu H, Fujimoto S, Hara S, Sato Y, Yamada K and Eto T: Relationship between serum $\operatorname{IgA} / \mathrm{C} 3$ ratio and progression of $\operatorname{Ig} \mathrm{A}$ nephropathy. Intern Med 43: 1023-1028, 2004.

27. Lee SM, Rao VM, Franklin WA, et al: IgA nephropathy: morphologic predictors of progressive renal disease. Hum Pathol 13: 314-322, 1982.

28. Glassock RJ: The treatment of IgA nephropathy: status at the end of the millenium. J Nephrol 12: 288-296, 1999.

29. Alexopoulos E: Treatment of primary IgA nephropathy. Kidney Int 65: 341-355, 2004.

30. Usui J, Yamagata K, Kai H, et al: Heterogeneity of prognosis in adult IgA nephropathy, especially with mild proteinuria or mild histological features. Intern Med 40: 697-702, 2001. 